\title{
A New Approach to Catastrophic Injury: Spinal Cord Injury Patients
}

\author{
S. I. Weingarden, MD, J. P. Kuric, RN, BSN, CCRN, J. G. Belen, MD, \\ P. M. Graham, RN, BA, CRRN. \\ Southeastern Michigan Spinal Cord Injury System Rehabilitation Institute, \\ Detroit Receiving Hospital/University Health Center, 261 Mack Blvd, Detroit, \\ Michigan 48201, USA.
}

\begin{abstract}
Summary
Catastrophic injuries and illnesses create great financial strains on patients who require lifetime care. Families, health care providers and insurers recognise that individual patient care needs require a closer look at the prudent allocation of health care benefit dollars. Blue Cross Blue Shield of Michigan has initiated an approach to this problem called 'case management'. Two ventilator dependent quadriplegics were discharged home utilising the case management concept. This system provides many advantages for patients. However, the health care team needs to be aware of the drawbacks of the system.
\end{abstract}

Key words: Spinal cord injury; Patient care planning; Long term care.

High cervical quadriplegia creates significant long term care needs after discharge from hospital. While some patients with private insurance, or financially strong family systems, are able to obtain the care and equipment which would allow return home, Medicare, Medicaid and Blue Cross Blue Shield programmes have been historically problematic. The limited number of home nursing or attendant care hours paid for by these programmes has precluded discharge home, and necessitated a prolonged hospital stay or nursing home discharge for the high quadriplegic patient.

Recently, Blue Cross Blue Shield of Michigan, in negotiations with various unions, developed a new concept for benefit adjudication. Described as a 'case management system', it has added greater flexibility to the traditional contracts. This approach has allowed 2 patients with ventilator dependent quadriplegia and limited family support to return home. In the process, advantages and pitfalls of the system have been learned. The intent of this paper is to describe our experiences and make suggestions for future refinements.

\section{Traditional coverage vs. cáse management}

A traditional Blue Cross Blue Shield plan covers 365 days of hospitalisation including surgery, treatment, and medications utilised by the patient in hospital. 
Table I

\begin{tabular}{lcc}
\hline \multicolumn{1}{c}{ Service } & Traditional plan & Case management \\
\hline 24 hour nursing care & Not covered & Covered \\
Home modifications & Not covered & Covered \\
Heavy equipment & Not covered & Covered \\
\hline
\end{tabular}

Table II Patient X

\begin{tabular}{lc}
\hline $\begin{array}{l}\text { Acute care for 20 days } \\
\text { Total Hospital Cost }\end{array}$ & $\begin{array}{c}\text { Rehabilitation for } 60 \text { days }=80 \text { days total. } \\
\text { Average per Day Cost }\end{array}$ \\
& $\begin{array}{c}\$ 150,576 \cdot 00 \\
1,882 \cdot 20\end{array}$ \\
& $\$ 150,576 \cdot 00$ \\
Days Available & $80 \quad \$ 1,882 \cdot 20$ \\
Banked Account & $365-80=285$ \\
\hline
\end{tabular}

Following discharge, this plan allows for intermittent skilled care of short duration (i.e. 2-3 visits per week for 6 weeks). Skilled care indicates that the care provided must be given by a health professional with special knowledge and skills. The traditional plan does not cover 24 hour nursing care, home modifications, or heavy equipment such as ventilators, etc. The contract is set and no modifications can be made for individual care.

The Blue Cross Blue Shield of Michigan case management system was introduced on 1 June 1986. The expressed intent of the programme was to 'provide high quality, cost effective, alternative treatment options for patients with catastrophic, chronic, terminal or long term illnesses. Most often the programme will facilitate leaving the hospital to recover at home, or in a less intensive environment' (Bearden, 1986). The case management approach allows unused hospital days to be converted into a dollar amount 'banked' for an individual patient application and use. The money can be utilised for 24 hour nursing care, home modifications, and heavy equipment (see Table I).

An example of this system is as follows: A patient is hospitalised under a traditional contract. After 20 days of acute care and 60 days of rehabilitation, the patient is ready for discharge but still requires skilled home care services in order to return home safely. The average per day cost for this specific hospitalisation is multiplied by the remaining number of available days under the contract (see Table II). The resultant dollar value becomes a 'banked account' or 'pool' the patient may 'drain'. A detailed care plan is developed by the Blue Cross case manager prior to discharge. This plan defines the equipment, home modifications, and nursing needs that these funds will purchase. Negotiations between the case manager, patient, family, physician and staff are possible. The goal is to prolong and maximise benefits to the patient's advantage.

Additional services and benefits not normally covered by Blue Cross Blue Shield policies but essential for the individual's home care can be provided under this system. All these, however, must be part of the medical treatment plan as determined by the patient's physician and must be considered accepted medical practice.

Most professionals realise that the amount billed to Blue Cross is not the 
amount that is reimbursed to the hospital; therefore, the 'banked pool' is usually reduced (according to a formula) to reflect what Blue Cross would have 'paid' for the hospitalisation and not the actual costs.

\section{Case report 1}

A 25-year-old male was admitted to hospital after being shot through the nose. The bullet traversed the spinal cord just below the foramen magnum, and the patient only survived because of immediate CPR given by a bystander. The patient was diagnosed as a $\mathrm{C} 1$ ventilator dependent quadriplegic.

The patient's hospitalisation was prolonged by numerous complications. These included a GI bleed, repeated episodes of pneumonia, urinary tract infections, recurrent nausea and vomiting, and sepsis. The neurologic status remained unchanged with the patient only being able to be off full ventilatory support for a few seconds at a time.

Inpatient rehabilitation hospitalisation was not an option as this would quickly reduce the amount of the 'banked pool' available for ongoing care. Because of this, throughout the acute hospital stay the patient was involved in a limited but continuing rehabilitation programme. This programme included bladder and bowel management, face, mouth and tongue strengthening, extremity and chest wall range of motion, progressive sitting, and ordering of durable medical equipment. The cuffed tracheostomy tube was changed to a Jackson tube which allowed verbal communication by the patient. The bedside ventilator was replaced with a portable LP6 ventilator (Life Care), which in conjunction with a portable 12-volt dry cell battery allowed the patient wheelchair mobility.

The patient's mother was the person most appropriate to provide for his care. Although the mother wanted the patient to return to her home, she did not feel capable of caring for him. The patient adamantly refused nursing home placement. His mother's home required modifications for wheelchair access, and additional living space for the patient and his care givers.

After lengthy discussions, it was agreed to pursue the Blue Cross case management programme. A care plan was developed with provisions for the necessary home modifications and equipment. Allowances were made for outpatient rehabilitation and followup medical care. With the remaining dollars, 24 hour per day home nursing care was purchased. LPNs rather than RNs were used to reduce cost and thus extend the duration of home care services. This was determined to be approximately 26 months.

During the planning phase, the patient's mother and the patient were instructed in all aspects of the patient's care. After 99 days of hospitalisation, the patient was discharged home. He was then enrolled in the day programme for ventilator dependent quadriplegic outpatients. Other than a short 2 day rehospitalisation for viral pneumonitis the patient continues to do well.

\section{Case report 2}

A 44-year-old male sustained a high cervical spinal cord injury following a fall. The patient was a ventilator dependent quadriplegic. A medical, pulmonary, and rehabilitation programme similar to Case 1 was followed.

The patient's only close family member was a married brother. While he did not feel capable of caring for him, he strongly felt that he wanted to bring his brother home and avoid nursing home placement.

The patient was eligible for the Blue Cross case management programme. It was decided by the patient and his brother to pursue this option. An approach similar to the above case was pursued. Some home modifications were done by the brother to save 'banked dollars' for future home care needs.

Following 144 days of hospitalisation the patient was discharged home. However, due to lower average per day hospital costs and a longer hospital stay (by 45 days), a much shorter duration of home nursing care was projected. The family understood that any care provided by the family would further extend his benefits. 


\section{Discussion}

Disposition of ventilator dependent quadriplegic patients requiring 24 hour per day care has been difficult under the best of conditions. Blue Cross covered patients had previously not fared well in this regard and prolonged hospitalisation or nursing home placement frequently was the only option. No flexibility was present in utilising covered benefits in alternative ways even if both the patient and/or Blue Cross would benefit.

The case management approach was first introduced by Medicare as a demonstration project in 1974 and by Medicaid in 1981. The programme was intended to co-ordinate health care for the elderly from diverse providers and suppliers (Omenn and Ehreth, 1986; Quinn, 1986). The involvement of a case manager who co-ordinated the benefit and the provision of alternative care programmes (e.g. home care instead of nursing home) was estimated to cut costs by $30 \%$ under one programme (Quinn, 1986).

The case management programme approach consists of four steps: (1) Assessment and planning to determine the scope of services and equipment needed, (2) co-ordination and referral, (3) treatment of medical problems and, (4) continued monitoring to assess progress (Merrill, 1985).

The programme as defined by Blue Cross Blue Shield of Michigan is somewhat different from the original concept. The goal remains the same; namely, a customised plan for patient care utilising alternative approaches, that allows for maximal use of patient contract benefits (Goulet, 1986).

The case management programme as described in this report offers obvious benefits. Advantages to the patient include reduction in the length of hospitalisation, avoidance of nursing home placement and discharge to a home with all necessary modifications, equipment and home care services. The system, in fact, benefits the carrier as well. Patient dollars that would have been spent rapidly with continued intensive care unit hospitalisation are disbursed at a slower rate by the company.

Although the 'pooled' dollars are finite and will be exhausted after a period of time, the additional time obtained permits planning for the development of alternative arrangements. This includes Medicare, Medicaid and even private fund raising.

Although the programme is in its initial phase, some pitfalls have already been noted. The longer the initial hospitalisation the less the banked account will hold for the patient upon discharge. Since the dollar value of the account is determined by a multiplier of the average per day cost, the amount will vary. The greater the hospital costs per day (i.e. surgery, IVs, monitors, etc.) the greater the benefit to the patient. The implication is that an expensive stay will maximise the patient's benefits.

With rehospitalisation, the programme calls for recalculating the benefits. The cost of the hospitalisation is subtracted from the 'banked pool'. Also, the average per day cost of the most recent hospitalisation becomes the new multiplier for calculating the size of the fund. This approach created a problem for Case 1. His short term rehospitalisation did not require high cost services or procedures. Since the costs per day during this hospitalisation were much less than during his first stay, the multiplier was reduced. He thus lost a number of 
days of future home care secondary to the dollars actually spent during the hospitalisation, as well as to the recalculated lower value.

Interestingly, care has been impeded by the patient's desire to keep costs down. Repeat medical visits require expensive transportation besides the costs of the visit. As a result, there has been reluctance to expend these funds and a reliance on the telephone even when not appropriate. A mechanism to insure appropriate expenditures requires to be developed.

\section{Summary}

The Blue Cross Case Management programme allows more flexibility in providing options for the catastrophically injured patient. These options were not available under the traditional coverage plans. As health professionals, it is our responsibility to become knowledgeable of resources available to our patients and how to effectively utilise these resources to benefit the patient.

Under the present programme, a consolidated short term hospitalisation with procedures and services provided to increase the per day cost is the way to maximise benefits. Ironically, keeping costs down are detrimental to the patient's long term care needs. Inexpensive rehospitalisations should be avoided by the patient, even when appropriate, in order to maintain the dollar benefit. It is hoped that this provision will be modified by future negotiations between Blue Cross Blue Shield and the subscriber groups.

\section{Acknowledgement}

We wish to thank Ms Mary Price for her word processing skills and patience in preparing this manuscript.

\section{References}

BeARDEN DG 1986 Blue Cross Blue Shield of Michigan. Memorandum. Goulet C 1986 Blue Cross Blue Shield. Alternate Delivery Systems Health Matrix 4(4):7-11.

Merrill JC 1985 Defining Case Management. Business Health 2(8):5-9.

OMENN GS, EHRETH J 1986 A New Look for Medicaid. Business and Health 4(2):7-11.

QuINN J 1986 Seeking Quality Alternatives in the Community. Business and Health 3(8):21-24. 